\title{
HOLOMORPHIC MAPS WHICH PRESERVE INTRINSIC METRICS OR MEASURES
}

\author{
IAN GRAHAM
}

\begin{abstract}
Suppose that $M$ is a domain in a taut complex manifold $M^{\prime}$, and that $\Omega$ is a strictly convex bounded domain in $C^{n}$. We consider the following question: given a holomorphic map $F: M \rightarrow \Omega$ which is an isometry for the infinitesimal Kobayashi metric at one point, must $F$ be biholomorphic? With an additional technical assumption on the behavior of the Kobayashi distance near points of $\partial M$, we show that $F$ gives a biholomorphism of $M$ with an open dense subset of $\Omega$. Moreover, $F$ extends as a homeomorphism from a larger domain $\widetilde{M}$ to $\Omega$. We also give some related results-refinements of theorems of Bland and Graham and Fornaess and Sibony, and the answer to a question of Graham and Wu.
\end{abstract}

\section{INTRODUCTION AND STATEMENT OF RESULTS}

The main purpose of this paper is to prove the following theorem:

Theorem 3.12. Let $M$ be a domain in a taut complex manifold $M^{\prime}$. Let $\Omega$ be a strictly convex bounded domain in $\mathbf{C}^{n}$. Let $F: M \rightarrow \Omega$ be a holomorphic map which is an isometry for the infinitesimal Kobayashi metric at a point $p \in$ $M$. Suppose that the pair $\left(M, M^{\prime}\right)$ satisfies the following condition: whenever $\tilde{p} \in \partial M$ is such that there exists a sequence $\left\{p_{j}\right\} \subset M$ such that $p_{j} \rightarrow \tilde{p}$ and $K_{M}\left(p, p_{j}\right)$ is bounded, then for all $\varepsilon>0$ there exists a neighborhood $U$ of $\tilde{p}$ such that $K_{M}\left(q_{1}, q_{2}\right)<\varepsilon$ whenever $q_{1}, q_{2} \in M \cap U$. Then there is a domain $\widetilde{M}$ such that $M \subset \widetilde{M} \subset \bar{M}$, and a homeomorphism $\widetilde{F}: \widetilde{M} \rightarrow \Omega$ which extends $F$. In particular $M$ is biholomorphic to an open dense subset of $\Omega$.

The open dense subset of $\Omega$ evidently must have the property that the inclusion map $i: F(M) \rightarrow \Omega$ is a Kobayashi isometry at one point. This could occur, for example, if $\Omega \backslash F(M)$ is a complex analytic subvariety of codimension two (see $[4,5]$ and Proposition 3.13). In this case $\widetilde{F}$ is actually biholomorphic-it would be of interest to prove this in general. Theorem 3.12 generalizes the main result of [12], in which $M$ itself is assumed to be taut.

We also give some related results. First we improve the hypotheses in a theorem of Bland and Graham [2, Theorem 2(d)], concerning the existence of

Received by the editors September 15, 1988.

1980 Mathematics Subject Classification (1985 Revision). Primary 32H20.

Partially supported by the Natural Sciences and Engineering Research Council of Canada under grant A9221. 
holomorphic maps which are Kobayashi isometries at one point ( $\S 4)$. Next we recast a theorem of Fornaess and Sibony [8, Theorem 3.2] in a form which makes an assumption on the Eisenman-Kobayashi volume form rather than on the Kobayashi metric ( $\S 5)$. Finally we answer (in the negative) a question of Graham and $\mathrm{Wu}$ [13] concerning the concept of measure-hyperbolic completeness - a measure-hyperbolically complete domain need not be a domain of holomorphy.

The historical antecedents of Theorem 3.12 are by now fairly numerous. $\mathrm{H}$. Cartan's classical theorem on fixed points of holomorphic self-maps of bounded domains was generalized to hyperbolic manifolds in the late 1960's (S. Kobayashi [16, Chapter V, Theorem 3.3], H. Wu [29, Theorems C and $\left.C^{\prime}\right]$, W. Kaup [15, Satz 3.6]). In particular a holomorphic self-map of a hyperbolic manifold, which is an isometry for the infinitesimal Kobayashi metric at a fixed point, must be biholomorphic. If one moves away from self-maps with fixed points, and considers instead a holomorphic mapping $F: M_{1} \rightarrow M_{2}$ between complex manifolds, then the assumption that $F$ be an isometry for the Caratheodory or Kobayashi metric, even at all points, need not imply that $F$ is biholomorphic ([27]; also Remark 3.15). Several positive results have been obtained in recent years using the work of Lempert [18-20]-see Vigue $[27,28]$, Graham and $\mathrm{Wu}$ [14], Patrizio [21], and Graham [12]. These results require that one or both of $M_{1}$ and $M_{2}$ should be bounded convex domains in $\mathrm{C}^{n}$. For example, Patrizio [21, Theorem 3.1], generalizing a result of Graham and $\mathrm{Wu}$ [14], showed that a holomorphic map between strictly convex domains which is a Kobayashi/Carathéodory isometry at one point is biholomorphic. Vigué, meanwhile, had shown that a map from a convex bounded domain to an arbitrary bounded domain, which is a Carathéodory isometry at one point, must be biholomorphic [27, Théorème 3.1]. The author recently showed that a holomorphic map from a taut complex manifold to a strictly convex bounded domain, which is a Kobayashi isometry at one point, must be biholomorphic [12]. Vigué also has a result for mappings into convex domains [28, Théorème 4.3]. Finally it should be mentioned that a generalization of $\mathrm{H}$. Cartan's theorem has recently been obtained by Burns and Krantz [3], under the assumption that the fixed point lies in the boundary.

The author would like to express his appreciation to the Mathematics Department at Stanford University for its warm hospitality during his sabbatical year, when this paper was written.

\section{DEFINITIONS AND PRELIMINARIES}

We begin by recalling the definition of the infinitesimal Kobayashi pseudometric. Let $M$ be a connected complex manifold. Let $D$ be the unit disc in C. If $p \in M$, and $\xi \in T_{p} M$, we define

$$
\begin{aligned}
& K(p ; \xi)=\inf \left\{|v| \mid v \in T_{0} D\right. \text { and there exists a holomorphic } \\
& \left.\qquad \operatorname{map} f: D \rightarrow M \text { such that } f(0)=p \text { and } d f_{0}(v)=\xi\right\} .
\end{aligned}
$$


The Kobayashi pseudo-distance between two points $p$ and $q$ we denote by $K(p, q)$. It is obtained by integrating $K(;)$ over piecewise-smooth curves joining $p$ to $q$ and taking the infimum.

$M$ is called hyperbolic if $K(p, q)>0$ whenever $p \neq q . M$ is called taut [29] if whenever $N$ is a complex manifold and $f_{j}: N \rightarrow M$ is a sequence of holomorphic mappings, then either there exists a subsequence which converges uniformly on compact subsets to a holomorphic map $f: N \rightarrow M$, or a subsequence which is compactly divergent. If this condition holds when $N=D$ then $M$ is already taut [1]. If $M$ is taut then it is hyperbolic, and the infimum in (2.1) is assumed for some mapping $f$.

We shall usually omit the phrase "uniformly on compact subsets" when discussing convergence of a sequence of holomorphic mappings $f_{j}: N \rightarrow M$, when it is clear that we are dealing with this type of convergence. Strictly speaking we must choose a metric on $M$ compatible with its topology before talking about uniform convergence on compact subsets. However the resulting topology on the set of holomorphic mappings from $N$ to $M$ is independent of this choice of metric, and is simply the compact-open topology. Also Latin subscripts $j$, $k, l$, etc. will always range over the positive integers, i.e. will be used to index sequences, without our repeating this fact each time.

Let us also recall that the infinitesimal Carathéodory pseudo-metric is defined by

$$
C(p ; \xi)=\sup \left\{\left|d g_{p}(\xi)\right| \mid g: M \rightarrow D \text { holomorphic, } g(p)=0\right\} \text {. }
$$

The Caratheodory pseudo-distance between two points $p$ and $q$ is defined by

$$
C(p, q)=\sup \{\rho(g(p), g(q)) \mid g: M \rightarrow D \text { holomorphic }\}
$$

where $\rho$ is the Poincare distance, i.e.

$$
\rho\left(z_{1}, z_{2}\right)=\frac{1}{2} \ln \left(1+\left|\frac{z_{1}-z_{2}}{1-\bar{z}_{1} z_{2}}\right|\right)-\frac{1}{2} \ln \left(1-\left|\frac{z_{1}-z_{2}}{1-\bar{z}, z_{2}}\right|\right), \quad z_{1}, z_{2} \in D .
$$

Integrating $C(;)$ along curves leads to a pseudo-distance $\widetilde{C}(p, q)$ such that $\widetilde{C}(p, q) \geq C(p, q) . \quad M$ is called Carathéodory-hyperbolic if $C(p, q)>0$ whenever $p \neq q$.

We shall use subscripts to indicate explicitly the manifold on which we are considering these metrics, e.g. $K_{M}(p ; \xi)$. A holomorphic map $F: M \rightarrow N$ is a Kobayashi isometry at a point $p \in M$ if $K_{M}(p ; \xi)=K_{N}\left(F(p) ; d F_{p}(\xi)\right)$ for all $\xi \in T_{p} M$, i.e. if $d F_{p}$ preserves the infinitesimal Kobayashi metric. (In general this is not the same as requiring that $F$ preserve the Kobayashi distance to first order at $p$. But it is the same if $M$ is taut and the Kobayashi indicatrix at $p$ is convex. See [2] and the references there.) Similarly $F$ is a Caratheodory isometry at $p$ if $C_{M}(p ; \xi)=C_{N}\left(F(p), d F_{p}(\xi)\right)$ for all $\xi \in T_{p} M$. (This is the same as requiring that $F$ preserve the Carathéodory distance to first order at p.)

Next we recall a definition of Vesentini [25]. A holomorphic map $\phi: D \rightarrow M$ is a complex geodesic for the Carathéodory metric if $\phi$ is an isometry for 
$C($, ), i.e.

$$
C_{M}\left(\phi\left(z_{1}\right), \phi\left(z_{2}\right)\right)=C_{D}\left(z_{1}, z_{2}\right)=\rho\left(z_{1}, z_{2}\right)
$$

for all $z_{1}, z_{2} \in D$. Similarly $\phi$ is a complex geodesic for the Kobayashi metric if

$$
K_{M}\left(\phi\left(z_{1}, \phi\left(z_{2}\right)\right)=K_{D}\left(z_{1}, z_{2}\right)=\rho\left(z_{1}, z_{2}\right)\right.
$$

for all $z_{1}, z_{2} \in D$.

If $M$ is a convex bounded domain in $\mathbf{C}^{n}$ then the Carathéodory and Kobayashi metrics coincide, and so do the complex geodesics. In fact the complex geodesics are precisely the stationary maps studied in great detail by Lempert [18-20]. (Royden and Wong extended this concept from the smoothly bounded case to the general convex case [24].)

We shall usually denote a convex bounded domain in $\mathbf{C}^{n}$ by $\Omega$. Such a domain is strictly convex if whenever $L$ is a closed line segment such that $L \subset \bar{\Omega}$ we have $\stackrel{\circ}{L} \subset \Omega$. For a strictly convex domain, the map realizing the infimum in (2.1) is unique (provided one assumes that $d f_{0}\left(\frac{d}{d z}\right)$ is a positive multiple of $\xi$ ), and it is a stationary map. Also, given two points $p, q \in \Omega$ there is a unique stationary map $\phi: D \rightarrow \Omega$ such that $\phi(0)=p$ and $\phi(r)=q$ for some $r>0$ [18].

In order to deal with the situation of Theorem 3.12 we need the following extension of the concept of complex geodesic:

Definition 2.2. Suppose $M$ is a domain in a complex manifold $M^{\prime}$. A holomorphic map $\phi: D \rightarrow \bar{M}$ is called a complex geodesic for $K_{M}$ in $\bar{M}$ (resp. for $C_{M}$ in $\left.\bar{M}\right)$ if

(i) $\phi(D) \cap M$ is nonempty;

(ii) $\phi$ is the uniform limit on compact subsets of a sequence of holomorphic mappings $f_{k}: D \rightarrow M$;

(iii) $K_{M}\left(\phi\left(z_{1}\right), \phi\left(z_{2}\right)\right)=K_{D}\left(z_{1}, z_{2}\right)=\rho\left(z_{1}, z_{2}\right)\left(\right.$ resp. $C_{M}\left(\phi\left(z_{1}\right), \phi\left(z_{2}\right)\right)=$ $\left.C_{D}\left(z_{1}, z_{2}\right)=\rho\left(z_{1}, z_{2}\right)\right)$ whenever $\phi\left(z_{1}\right), \phi\left(z_{2}\right) \in M$.

Of course condition (iii) implies that $d \phi_{z}$ is an isometry for $K(;)$ (resp. for $C(;))$ whenever $\phi(z) \in M$.

The technical condition on certain boundary points of $M$ in Theorem 3.9 we shall call condition $T$ :

Definition 2.3. Let $M$ be a domain in a complex manifold $M^{\prime}$. The pair $\left(M, M^{\prime}\right)$ satisfies condition $T$ if whenever $\tilde{p} \in \partial M$ is such that there exists $\left\{p_{j}\right\}_{j=1}^{\infty} \subset M$ and $p \in M$ with $p_{j} \rightarrow \tilde{p}$ and $K\left(p, p_{j}\right)$ bounded, then for all $\varepsilon>0$ there exists a neighborhood $U$ of $\tilde{p}$ in $M^{\prime}$ such that $K_{M}\left(q_{1}, q_{2}\right)<\varepsilon$ for all $q_{1}, q_{2} \in M \cap U$.

We note that the point $p \in M$ plays no special role-if $\left\{K\left(p, p_{j}\right)\right\}$ is bounded then so is $\left\{K\left(p^{\prime}, p_{j}\right)\right\}$ for any other point $p^{\prime} \in M$.

These are all the preliminaries needed for the main theorem. However Theorems 4.1 and 5.1 require a few additional definitions concerning intrinsic 
measures. Let $p$ be a point in a complex manifold of dimension $n$ and let $z_{1}, \ldots, z_{n}$ be local coordinates in a neighborhood of $p$. Let $B^{n}$ denote the unit ball in $\mathbf{C}^{n}$. The Eisenman-Kobayashi volume density is defined by

$$
\begin{aligned}
E_{n}( & \left.p \frac{\partial}{\partial z_{1}} \wedge \cdots \wedge \frac{\partial}{\partial z_{n}}\right) \\
& =\inf \left\{\frac{1}{|J f(0)|^{2}} \mid f: B^{n} \rightarrow M, f \text { holomorphic, } f(0)=p\right\} .
\end{aligned}
$$

We also define, whenever $c \in \mathbf{C}$,

$$
E_{n}\left(p ; c \frac{\partial}{\partial z_{1}} \wedge \cdots \wedge \frac{\partial}{\partial z_{n}}\right)=|c|^{2 n} E_{n}\left(p ; \frac{\partial}{\partial z_{1}} \wedge \cdots \wedge \frac{\partial}{\partial z_{n}}\right)
$$

obtaining a pseudo-norm on $\Lambda^{n} T_{p} M$. The Eisenman-Kobayashi volume form is defined (invariantly) by

$$
\tau(p)=E_{n}\left(p ; \frac{\partial}{\partial z_{1}} \wedge \cdots \wedge \frac{\partial}{\partial z_{n}}\right)\left(\frac{i}{2}\right)^{n} d z_{1} \wedge d \bar{z}_{1} \wedge \cdots \wedge d z_{n} \wedge d \bar{z}_{n} .
$$

If $S$ is a Borel subset of $M$ then its Eisenman-Kobayashi volume is

$$
E_{n}(S)=\int_{S} \tau
$$

Similar constructions are used to define the Carathéodory volume density, the Carathéodory volume form, and the Carathéodory volume of a set $[7,10]$.

If $M$ is hyperbolic and of dimension $n$, we will denote the top-dimensional Hausdorff measure associated to the Kobayashi metric by $H_{K}^{2 n}$. If $z_{1}, \ldots, z_{n}$ are local coordinates in a neighborhood of $p$, we denote Lebesgue measure in these coordinates by $\lambda^{2 n}$. The density of $H_{K}^{2 n}$, or equivalently the RadonNikodym derivative of $H_{K}^{2 n}$ with respect to $\lambda^{2 n}$ we denote by $d H_{K}^{2 n} / d \lambda^{2 n}$. Evaluating at $p$ gives $\left(d H_{K}^{2 n} / d \lambda^{2 n}\right)(p)$. In general we have the inequality (see [2])

Finally, in $\S 6$ we need the following definitions.

$$
\frac{d H_{K}^{2 n}}{d \lambda^{2 n}}(p) \leq E_{n}\left(p ; \frac{\partial}{\partial z_{1}} \wedge \cdots \wedge \frac{\partial}{\partial z_{n}}\right) .
$$

Definition 2.4. A domain $G \varsubsetneqq \mathbf{C}^{n}$ is measure-hyperbolically complete at a point $p \in \partial G$ if whenever $U$ is a neighborhood of $p$ we have

$$
\int_{U \cap G} \tau_{G}=\infty \text {. }
$$

Definition 2.5 [13]. A domain $G \varsubsetneqq \mathbf{C}^{n}$ is measure-hyperbolically complete if it is measure-hyperbolically complete at all boundary points.

\section{HOLOMORPHIC MAPS INTO STRICTLY CONVEX DOMAINS}

In this section we give the proof of Theorem 3.12. The idea is to "pull back" the complex geodesics for the Kobayashi metric from $\Omega$ to $M$. Since $M$ is 
not taut, but is contained in a larger taut complex manifold, we obtain a family of holomorphic mappings from $D$ into $\bar{M}$. The assumption on the Kobayashi metric of $M$ near certain boundary points (condition $T$ ) is needed to guarantee that these mappings, called complex geodesics for $K_{M}$ in $\bar{M}$, are 1-1. Other properties of extremal discs in strictly convex domains, established by Lempert, are shown to hold for these complex geodesics. A map from a subset $\widetilde{M}$ of $\bar{M}$ onto $\Omega$ is defined by means of the correspondence of complex geodesics for $K_{M}$ in $\bar{M}$ and complex geodesics in $\Omega$. This map is easily seen to be $1-1$; however, to prove that it is a homeomorphism again requires condition $T$. Finally it is shown that $\widetilde{M} \supset M$. The case where $M$ itself is taut was dealt with in [12].

Lemma 3.1. Let $M$ be a domain in a complex manifold $M^{\prime}$. Suppose $\phi_{j}: D \rightarrow$ $\bar{M}, j=1,2, \ldots$, is a sequence of complex geodesics for $K_{M}$ in $\bar{M}$ (resp. for $C_{M}$ in $\bar{M}$ ) which converge to a holomorphic map $\phi: D \rightarrow \bar{M}$. Suppose that $\phi(D) \cap M$ is nonempty. Then $\phi$ is a complex geodesic for $K_{M}$ in $\bar{M}$ (resp. for $C_{M}$ in $\bar{M}$ ).

Proof. It is clear that $\phi$ is the uniform limit on compact subsets of a sequence of holomorphic maps $f_{k}: D \rightarrow M$. Suppose $\phi\left(z_{1}\right), \phi\left(z_{2}\right) \in M$. Then $\phi_{j}\left(z_{1}\right)$, $\phi_{j}\left(z_{2}\right) \in M$ for $j$ sufficiently large. Since $K_{M}($,$) is continuous (resp. C_{M}($, is continuous) we have

$$
\rho\left(z_{1}, z_{2}\right)=K_{M}\left(\phi_{j}\left(z_{1}\right), \phi_{j}\left(z_{2}\right)\right) \rightarrow K_{M}\left(\phi\left(z_{1}\right), \phi\left(z_{2}\right)\right)
$$

(resp. $\left.\rho\left(z_{1}, z_{2}\right)=C_{M}\left(\phi_{j}\left(z_{1}\right), \phi_{j}\left(z_{2}\right)\right) \rightarrow C_{M}\left(\phi\left(z_{1}\right), \phi\left(z_{2}\right)\right)\right)$. Hence the conditions of Definition 2.2 are satisfied by $\phi$.

Corollary 3.2. Let $M$ be a domain in a taut complex manifold and let $p \in M$. The set of points in $M$ which can be joined to $p$ by a complex geodesic for $K_{M}$ in $\bar{M}$ (resp. by a complex geodesic for $C_{M}$ in $\bar{M}$ ) is closed in $M$.

Proof. Let $\left\{p_{j}\right\} \subset M$ and $\left\{r_{j}\right\} \subset D$ be sequences of points and let $\phi_{j}: D \rightarrow \bar{M}$, $j=1,2, \ldots$, be complex geodesics for $K_{M}$ in $\bar{M}$ such that $\phi_{j}(0)=p$ and $\phi_{j}\left(r_{j}\right)=p_{j}$. Suppose that $p_{j} \rightarrow p^{\prime} \in M$. Since $K_{M}\left(p, p^{\prime}\right)<\infty$, the $r_{j}$ belong to a compact subset of $D$, hence a subsequence $r_{j_{k}}$ converges to a number $r \in D$. By the tautness of $M^{\prime}$ a subsequence of the $\phi_{j_{k}}$, which we shall again denote by $\phi_{j_{k}}$, converges to a holomorphic map $\phi: D \rightarrow \bar{M}$. Since $\phi(0)=p, \phi(D) \cap M$ is nonempty, so $\phi$ is a complex geodesic for $K_{M}$ in $\bar{M}$. Evidently $\phi(r)=\lim _{k \rightarrow \infty} \phi_{j_{k}}\left(r_{j_{k}}\right)=p^{\prime}$.

An identical argument applies to the case of complex geodesics for $C_{M}$ in $\bar{M}$.

Lemma 3.3. Let $M$ be a domain in a taut complex manifold $M^{\prime}$. Suppose that $\Omega$ is a strictly convex bounded domain in $\mathbf{C}^{n}$, and that $F: M \rightarrow \Omega$ is a holomorphic map which is an isometry for the infinitesimal Kobayashi metric at 
$p \in M$. Let $\xi \in T_{p} M$ and suppose that $\xi \neq 0$. Then there is a unique complex geodesic $\phi$ for $K_{M}$ in $\bar{M}$ such that $\phi(0)=p$ and $d \phi_{0}\left(\frac{d}{d z}\right)$ is a positive multiple of $\xi$.

Proof. Let $f_{k}: D \rightarrow M$ and $v_{k} \in T_{0} D$ be sequences of holomorphic maps and tangent vectors respectively, such that $f_{k}(0)=p, d f_{k}\left(v_{k}\right)=\xi,\left(d f_{k}\right)_{0}\left(\frac{d}{d z}\right)$ is a positive multiple of $\xi$, and $\left|v_{k}\right| \rightarrow K_{M}(p ; \xi)$. In view of the tautness of $M^{\prime}$ there is a subsequence $f_{k_{l}}$ and a holomorphic map $\phi: D \rightarrow \bar{M}$ such that $f_{k_{l}} \rightarrow \phi$. It is clear that $\phi(0)=p$ and that $d \phi_{0}\left(\frac{d}{d z}\right)$ is a positive multiple of $\xi$. Now using the tautness of $\Omega$ we obtain a further subsequence $f_{k_{l m}}$ and a holomorphic map $\psi: D \rightarrow \Omega$, such that $F \circ f_{k_{l m}} \rightarrow \psi$ and $\psi(0)=F(p)$. Since $F$ is a Kobayashi isometry at $p$ and since $\left|v_{k}\right| \rightarrow K_{M}(p ; \xi), \psi$ must be a Kobayashi isometry at 0 . Hence $\psi$ is a complex geodesic for $K_{\Omega}$ (or $C_{\Omega}$ ), because $\Omega$ is convex. The distance-decreasing property of $F$, together with the fact that $\phi=\lim f_{k_{1}}$, now implies that $K_{M}\left(\phi\left(z_{1}\right), \phi\left(z_{2}\right)\right)=\rho\left(z_{1}, z_{2}\right)$ whenever $\phi\left(z_{1}\right), \phi\left(z_{2}\right) \in M$. This shows that $\phi$ is a complex geodesic for $K_{M}$ in $\bar{M}$.

To show uniqueness, suppose that $\phi$ is any complex geodesic for $K_{M}$ in $\bar{M}$ such that $\phi(0)=p$ and $d \phi_{0}\left(\frac{d}{d z}\right)=\lambda \xi, \lambda>0$. Let $f_{k}: D \rightarrow M$ be a sequence of holomorphic maps such that $f_{k} \rightarrow \phi$. Of course $f_{k}(0) \rightarrow p$ and $\left(d f_{k}\right)_{0} \rightarrow d \phi_{0}$. Arguing as before, $\left\{F \circ f_{k}\right\}$ has a subsequence $\left\{F \circ f_{k_{l}}\right\}$ which converges to a map $\psi: D \rightarrow \Omega$. $\psi$ must be a complex geodesic such that $\psi(0)=F(p)$ and $\psi^{\prime}(0)=\lambda d F_{p}(\xi), \lambda>0$. These properties determine $\psi$ uniquely, for $\Omega$ is strictly convex. Hence $\phi$ is uniquely determined-near 0 this follows from the local invertibility of $F$, while elsewhere it follows from analytic continuation.

Remarks 3.4. (1) The passage to subsequences in the proof of Lemma 3.3 is actually not necessary. The uniqueness of $\phi$ under the stated conditions implies that $f_{k} \rightarrow \phi$ (in the first part of the proof), while the uniqueness of $\psi$ implies that $F \circ f_{k} \rightarrow \psi$ (in both parts of the proof).

(2) Using the fact that the Kobayashi and Carathéodory metrics coincide on $\Omega$, it can be seen that $F$ is a Carathéodory as well as a Kobayashi isometry at $p$. Hence $\phi$ must be a Carathéodory isometry at 0 . This implies, via an analog of [25, Proposition 3.2], that $\phi$ is a complex geodesic for $C_{M}$ as well as for $K_{M}$ in $\bar{M}$. Hence we may speak simply of complex geodesics in $\bar{M}$ (provided they pass through $p$ ).

Lemma 3.5. In the situation of Lemma 3.3, if in addition the pair $\left(M, M^{\prime}\right)$ satisfies condition $T$, then the following properties hold:

(a) If $\phi: D \rightarrow \bar{M}$ is a complex geodesic passing through $p$ then $\phi$ is 1-1.

(b) If $\phi_{1}$ and $\phi_{2}$ are complex geodesics in $\bar{M}$ such that $\phi_{1}(0)=\phi_{2}(0)=p$, then either $\phi_{1}(D) \cap \phi_{2}(D)=\{p\}$ or else there exists $\theta \in \mathbf{R}$ such that $\phi_{1}\left(e^{i \theta} z\right)=$ $\phi_{2}(z)$ for all $z \in D$. 
Proof. The idea is of course to deduce these results from the properties of complex geodesics in $\Omega$. It is clear that $\phi\left(z_{1}\right) \neq \phi\left(z_{2}\right)$ whenever $z_{1} \neq z_{2}$ and $\phi\left(z_{1}\right), \phi\left(z_{2}\right) \in M$. Let $f_{k}: D \rightarrow M$ be a sequence of holomorphic maps such that $f_{k} \rightarrow \phi$. We may assume that $\phi(0)=p$, and hence that $f_{k}(0) \rightarrow p$.

Let $\psi: D \rightarrow \Omega$ be the complex geodesic such that $F \circ f_{k} \rightarrow \psi$. Suppose that $\phi\left(z_{1}\right)=\phi\left(z_{2}\right) \in \partial M$ but that $z_{1} \neq z_{2}$. The sequence $\left\{f_{k}\left(z_{1}\right)\right\}$ is a sequence of points at bounded Kobayashi distance from $p$ converging to $\phi\left(z_{1}\right)$. Hence from condition $T$ with $\varepsilon=\frac{1}{2} K_{\Omega}\left(\psi\left(z_{1}\right), \psi\left(z_{2}\right)\right)$, we have

$$
K_{M}\left(f_{k}\left(z_{1}\right), f_{k}\left(z_{2}\right)\right)<\frac{1}{2} K_{\Omega}\left(\psi\left(z_{1}, \psi\left(z_{2}\right)\right)\right.
$$

for all sufficiently large $k$. By the distance-decreasing property of $F$, this implies

$$
K_{\Omega}\left(F \circ f_{k}\left(z_{1}\right), F \circ f_{k}\left(z_{2}\right)\right)<\frac{1}{2} K_{\Omega}\left(\psi\left(z_{1}\right), \psi\left(z_{2}\right)\right) .
$$

Hence, letting $k \rightarrow \infty$,

$$
K_{\Omega}\left(\psi\left(z_{1}\right), \psi\left(z_{2}\right)\right) \leq \frac{1}{2} K_{\Omega}\left(\psi\left(z_{1}\right), \psi\left(z_{2}\right)\right),
$$

a contradiction. This proves (a).

To prove (b), we let $\phi_{1}$ and $\phi_{2}$ be complex geodesics in $\bar{M}$ such that $\phi_{1}(0)=\phi_{2}(0)=p$. Let $f_{1 k}: D \rightarrow M$ and $f_{2 k}: D \rightarrow M$ be sequences of holomorphic maps such that $f_{1 k} \rightarrow \phi_{1}$ and $f_{2 k} \rightarrow \phi_{2}$. Of course $f_{1 k}(0) \rightarrow p$ and $f_{2 k}(0) \rightarrow p$. Let $\psi_{1}: D \rightarrow \Omega$ and $\psi_{2}: D \rightarrow \Omega$ be the complex geodesics such that $F \circ f_{1 k} \rightarrow \psi_{1}$ and $F \circ f_{2 k} \rightarrow \psi_{2}$. Then either there exists $\theta \in \mathbf{R}$ such that $\psi_{1}\left(e^{i \theta} z\right)=\psi_{2}(z)$ for all $z \in D$, or else $\psi_{1}(D) \cap \psi_{2}(D)=\{F(p)\}$. In the first case we have $\phi_{1}\left(e^{i \theta} z\right)=\phi_{2}(z)$ (in the first instance for $z$ near 0 , then for all $z \in D$ by analytic continuation). We wish to show that $\phi_{1}(D) \cap$ $\phi_{2}(D)=\{p\}$ in the second case. It is clear that if there exists $\tilde{p} \neq p$ such that $\tilde{p} \in \phi_{1}(D) \cap \phi_{2}(D)$, then $\tilde{p} \in \partial M$. Suppose there exists such a point, and let $z_{1}, z_{2} \in D$ be such that $\phi_{1}\left(z_{1}\right)=\phi_{2}\left(z_{2}\right)=\tilde{p}$. Then $\left\{f_{1 k}\left(z_{1}\right)\right\} \rightarrow \tilde{p}$ and

$$
K_{M}\left(p, f_{1 k}\left(z_{1}\right)\right) \leq \rho\left(0, z_{1}\right)+K_{M}\left(p, f_{1 k}(0)\right) .
$$

This shows that condition $T$ applies to the point $\tilde{p}$, since $K_{M}\left(p, f_{1 k}(0)\right) \rightarrow 0$ as $k \rightarrow \infty$. In particular, with $\varepsilon=\frac{1}{2} K_{\Omega}\left(\psi_{1}\left(z_{1}\right), \psi_{2}\left(z_{2}\right)\right)$ we obtain

$$
K_{M}\left(f_{1 k}\left(z_{1}\right), f_{2 k}\left(z_{2}\right)\right)<\frac{1}{2} K_{\Omega}\left(\psi_{1}\left(z_{1}\right), \psi_{2}\left(z_{2}\right)\right)
$$

for $k$ sufficiently large. The distance-decreasing property of $F$ gives

$$
K_{\Omega}\left(F \circ f_{1 k}\left(z_{1}\right), F \circ f_{2 k}\left(z_{2}\right)\right)<\frac{1}{2} K_{\Omega}\left(\psi_{1}\left(z_{1}\right), \psi_{2}\left(z_{2}\right)\right) .
$$

Letting $k \rightarrow \infty$ we obtain

$$
K_{\Omega}\left(\psi_{1}\left(z_{1}\right), \psi_{2}\left(z_{2}\right)\right) \leq \frac{1}{2} K_{\Omega}\left(\psi_{1}\left(z_{1}\right), \psi_{2}\left(z_{2}\right)\right),
$$

a contradiction. Q.E.D.

Lemmas 3.3 and 3.5 now make it possible to define an extension of $F$ under the assumptions of the theorem. We first define

$$
\widetilde{M}=\{\tilde{p} \in \bar{M} \mid \tilde{p} \text { can be joined to } p \text { by a complex geodesic in } \bar{M}\} \text {. }
$$


Now if $\tilde{p} \in \widetilde{M}$, let $\phi$ be a complex geodesic in $\bar{M}$ and let $z \in D$ be such that $\phi(0)=p, \phi(z)=\tilde{p}$. Let $f_{k}: D \rightarrow M$ be a sequence of holomorphic maps converging to $\phi$. Let $\psi: D \rightarrow \Omega$ be the complex geodesic in $\Omega$ such that $F \circ f_{k} \rightarrow \psi$. We now define $\widetilde{F}(\tilde{p})=\psi(z)$. Lemmas 3.3 and 3.5 show that $\widetilde{F}$ is well-defined. Also whenever $\phi$ and $\psi$ are related as just indicated (we will call them corresponding complex geodesics in $\bar{M}$ and $\Omega$ ), we have $\widetilde{F} \circ \phi=\psi$. $\widetilde{F}$ evidently gives a 1-1 mapping of $\widetilde{M}$ onto $\Omega$, in view of the known properties of complex geodesics in $\Omega$ [18].

We can also extend $K_{M}($,$) to a function \widetilde{K}($,$) defined on (M \times M) \cup$ $(\{p\} \times \widetilde{M}) \cup(\widetilde{M} \times\{p\})$ as follows:

$$
\left\{\begin{array}{c}
\widetilde{K}\left(p_{1}, p_{2}\right)=K_{M}\left(p_{1}, p_{2}\right) \text { whenever } p_{1}, p_{2} \in M ; \\
\widetilde{K}(p, \tilde{p})=\widetilde{K}(\tilde{p}, p)=\rho(0, z) \text { if there exists a complex } \\
\text { geodesic } \phi: D \rightarrow \bar{M} \text { such that } \phi(0)=p \text { and } \phi(z)=\tilde{p} .
\end{array}\right.
$$

Lemmas 3.3 and 3.5 show that this is well-defined. Note that we also have (with $p$ fixed and $\tilde{p}$ variable)

$$
\widetilde{K}(p, \tilde{p})=K_{\Omega}(F(p), \widetilde{F}(\tilde{p})) .
$$

We can also show

Lemma 3.9. Under the assumptions of Theorem 3.12 (in particular assuming condition $T), \widetilde{K}(p, \tilde{p})$ is continuous in $\tilde{p}$ for $\tilde{p} \in \widetilde{M}$.

Proof. If $\tilde{p} \in M$ this is known, so we may assume $\tilde{p} \in \partial M$. Suppose $\tilde{p}_{j} \in \widetilde{M}$ and $\tilde{p}_{j} \rightarrow \tilde{p}$. Let $\phi_{j}: D \rightarrow \bar{M}$ be complex geodesics and $r_{j} \in D, 0<r_{j}<1$ be points such that $\phi_{j}(0)=p, \phi_{j}\left(r_{j}\right)=\tilde{p}_{j}$. Let $\phi: D \rightarrow \bar{M}$ be a complex geodesic and $r \in D, 0<r<1$ be a point such that $\phi(0)=p, \phi(r)=\tilde{p}$. Let $f_{j k}: D \rightarrow M$ and $f_{k}: D \rightarrow M$ be holomorphic maps such that $f_{j k} \rightarrow \phi_{j}$ and $f_{k} \rightarrow \phi$ as $k \rightarrow \infty$. Then as $k \rightarrow \infty, f_{j k}\left(r_{j}\right) \rightarrow \tilde{p}_{j}$ and $f_{k}(r) \rightarrow \tilde{p}$, while $f_{j k}(0) \rightarrow p$ and $f_{k}(0) \rightarrow p$. Using the fact that $F \circ f_{j k} \rightarrow \tilde{F} \circ \phi_{j}$ (a complex geodesic in $\Omega$ ) and the distance-decreasing property of $F$, we see that $K_{M}\left(f_{j k}(0), f_{j k}\left(r_{j}\right)\right) \rightarrow \rho\left(0, r_{j}\right)$ as $k \rightarrow \infty$. This easily implies that $K_{M}\left(p, f_{j k}\left(r_{j}\right)\right) \rightarrow \rho(0, r)$ as $k \rightarrow \infty$. Similarly $K_{M}\left(p, f_{k}(r)\right) \rightarrow \rho(0, r)$ as $k \rightarrow \infty$. On the other hand condition $T$ applies to the point $\tilde{p}$, hence given $\varepsilon>0$ we have $K_{M}\left(f_{j k}\left(r_{j}\right), f_{l}(r)\right)<\varepsilon$ whenever $j, l$ are sufficiently large and $k \geq k(j)$. But this implies that $\rho\left(0, r_{j}\right) \rightarrow \rho(0, r)$. Q.E.D.

Lemma 3.10. Suppose that $\tilde{p}_{j} \in \widetilde{M}, j=1,2, \ldots$, and that $\tilde{p}_{j} \rightarrow \tilde{p} \in \widetilde{M}$ as $j \rightarrow \infty$. Suppose also that $\tilde{p} \neq p$. Let $\phi_{j}$ be the unique complex geodesic in $\bar{M}$ such that $\phi_{j}(0)=p$ and $\phi_{j}\left(r_{j}\right)=\tilde{p}_{j}$ for some number $r_{j}, 0<r_{j}<1$. Also let $\phi$ be the unique complex geodesic such that $\phi(0)=p$ and $\phi(r)=\tilde{p}$ for some number $r, 0<r<1$. Then $\phi_{j} \rightarrow \phi$ as $j \rightarrow \infty$.

Proof. Suppose not. Then since $M^{\prime}$ is taut there is a subsequence $\phi_{j_{k}}$ and a holomorphic map $\tilde{\phi}: D \rightarrow \widetilde{M}, \tilde{\phi} \not \equiv \phi$, such that $\phi_{j_{k}} \rightarrow \tilde{\phi}$. By Lemma $3.1 \tilde{\phi}$ is a 
complex geodesic. By Lemma $3.9 \tilde{K}\left(p, \tilde{p}_{j}\right) \rightarrow \widetilde{K}(p, \tilde{p})$, i.e. $\rho\left(0, r_{j}\right) \rightarrow \rho(0, r)$, i.e. $r_{j} \rightarrow r$. It follows that $\phi_{j_{k}}\left(r_{j_{k}}\right) \rightarrow \tilde{\phi}(r)$. But since $\tilde{p}_{j_{k}} \rightarrow \tilde{p}$ we have $\tilde{\phi}(r)=\phi(r)=\tilde{p}$, which contradicts the uniqueness of $\phi$.

Remark 3.11. The complex geodesics of $\Omega$ also have this property.

Theorem 3.12. Let $M$ be a domain in a taut complex manifold $M^{\prime}$. Let $\Omega$ be a strictly convex bounded domain in $\mathbf{C}^{n}$. Let $F: M \rightarrow \Omega$ be a holomorphic map which is an isometry for the infinitesimal Kobayashi metric at one point. Suppose that the pair $\left(M, M^{\prime}\right)$ satisfies condition $T$. Then there is a domain $\widetilde{M}$ such that $M \subset \widetilde{M} \subset \bar{M}$, and a homeomorphism $\widetilde{F}: \widetilde{M} \rightarrow \Omega$ which extends $F$. In particular $M$ is biholomorphic to an open dense subset of $\Omega$.

Proof. We are now in a position to prove that $\widetilde{F}: \widetilde{M} \rightarrow \Omega$ is a homeomorphism. We have already observed that $\widetilde{F}$ is a $1-1$ map of $\widetilde{M}$ onto $\Omega$.

To show that $\widetilde{F}$ is continuous, let $\left\{\tilde{p}_{j}\right\}_{j=1}^{\infty} \subset \widetilde{M}$ and suppose that $\tilde{p}_{j} \rightarrow \tilde{p} \in$ $\widetilde{M}$. We may suppose that $\tilde{p} \neq p$, in fact since $F$ is continuous we may suppose that $\tilde{p} \in \widetilde{M} \backslash M$. Let $\phi_{j}: D \rightarrow \bar{M}$ be complex geodesics and $r_{j} \in D, r_{j}>0$ be points such that $\phi_{j}(0)=p, \phi_{j}\left(r_{j}\right)=\tilde{p}_{j}$. Also let $\phi: D \rightarrow \bar{M}$ be a complex geodesic and $r \in D, r>0$, be a point such that $\phi(0)=p, \phi(r)=\tilde{p}$. Let $\psi_{j}=\widetilde{F} \circ \phi_{j}, j=1,2, \ldots$, and $\psi=\widetilde{F} \circ \phi$ be the corresponding complex geodesics in $\Omega$. By Lemma 3.10, $\phi_{j} \rightarrow \phi$ as $j \rightarrow \infty$. Hence, by the continuity of $F, \psi_{j} \rightarrow \psi$ uniformly on a neighborhood of 0 . But this implies, by the tautness of $\Omega$, that $\psi_{j} \rightarrow \psi$ uniformly on compact subsets of $D$. Also by Lemma 3.9, $r_{j} \rightarrow r$ as $j \rightarrow \infty$. It therefore follows that $\psi_{j}\left(r_{j}\right) \rightarrow \psi(r)$, i.e. $\widetilde{F}\left(\tilde{p}_{j}\right) \rightarrow \widetilde{F}(\tilde{p})$. This shows that $\widetilde{F}$ is continuous.

To show that $\widetilde{F}^{-1}$ is continuous we essentially use the same argument, reversing the roles of $\widetilde{M}$ and $\Omega$. Let $\left\{q_{j}\right\}_{j=1}^{\infty} \subset \Omega$ and suppose $q_{j} \rightarrow q \in \Omega$. Let $\tilde{p}_{j}$ be the unique point in $\widetilde{M}$ such that $\widetilde{F}\left(\tilde{p}_{j}\right)=q_{j}$, and let $\tilde{p}$ be the unique point in $\widetilde{M}$ such that $\widetilde{F}(\tilde{p})=q$. We wish to show that $\tilde{p}_{j} \rightarrow \tilde{p}$. We may assume that $q \neq F(p)$ since $F$ is biholomorphic in a neighborhood of $p$. Let $\phi_{j}, \psi_{j}, \phi, \psi, r_{j}$ and $r$ be as in the previous paragraph. By Remark 3.11 we have $\psi_{j} \rightarrow \psi$. Since $F$ is biholomorphic in a neighborhood of $p$, this implies that $\phi_{j} \rightarrow \phi$ uniformly on some neighborhood of 0 . The tautness of $M^{\prime}$ now implies that $\phi_{j} \rightarrow \phi$ uniformly on compact subsets of $D$. Since $K_{\Omega}\left(F(p), q_{j}\right)=\rho\left(0, r_{j}\right)$ and $K_{\Omega}(F(p), q)=\rho(0, r)$ we have $r_{j} \rightarrow r$ as $j \rightarrow \infty$. It follows that $\phi_{j}\left(r_{j}\right) \rightarrow \phi(r)$, i.e. $\tilde{p}_{j} \rightarrow \tilde{p}$. We have thus shown that $\widetilde{F}$ is a homeomorphism.

It is clear that $\widetilde{M} \subset \bar{M}$, so it remains only to show that $\widetilde{M}$ contains $M$. $\widetilde{M} \cap M$ certainly contains a neighborhood of $p$, so it is nonempty. $\widetilde{M} \cap M$ is open since $\widetilde{M}$, being homeomorphic to $\Omega$, is open. On the other hand, Corollary 3.2 shows that $\widetilde{M} \cap M$ is closed in $M$. This is enough to conclude that $\widetilde{M} \cap M=M$, for we have assumed that $M$ is connected. This completes the proof. 
Examples which illustrate Theorem 3.12 may be constructed using the following:

Proposition 3.13. Let $G$ be a domain in $\mathbf{C}^{n}$, and let $A$ be a closed subset of $G$ which is of the first category in a nowhere dense closed analytic subset $V$ of $G$. Then the inclusion map $i: G \backslash A \rightarrow G$ is an isometry for the infinitesimal Kobayashi metric at all points.

Remark 3.14. The papers of Campbell and Ogawa [5] and Campbell, Howard and Ochiai [4] show that $i$ is an isometry for the Kobayashi distance. A separate (but similar) argument is needed to show that the infinitesimal Kobayashi metric is preserved. (When $G$ is taut the cited results imply, by taking directional derivatives of the Kobayashi distance, that the convex hull of the Kobayashi indicatrix at a point $p \in G \backslash A$ is the same for $K_{G}(p ;)$ and $K_{G \backslash A}(p ;)$. See [2].)

Proof of Proposition 3.13. Let $p \in G \backslash A$ and let $\xi \in T_{p} G$. Suppose that $f: D \rightarrow$ $G$ is holomorphic, $f(0)=p$, and $f^{\prime}(0)=c \xi$ where $c>0$. Let us also suppose for the moment that $f(D) \subset \subset G$. Consider the map $H: D^{*} \times V \rightarrow \mathbf{C}^{n}$ defined by $H(z, w)=z^{-2}(w-f(z))$. By [5, Lemma 1], $H\left(D^{*} \times A\right)$ is of the first category in $\mathbf{C}^{n}$. Hence there is a sequence $\left\{\varepsilon_{j}\right\}_{j=1}^{\infty}$ of points in $\mathbf{C}^{n}$ such that $\varepsilon_{j} \rightarrow 0$ as $j \rightarrow \infty$ and $\varepsilon_{j} \notin H\left(D^{*} \times A\right)$. But this means that the maps $f_{j}: D \rightarrow \mathbf{C}^{n}$ defined by $f_{j}(z)=f(z)+\varepsilon_{j} z^{2}$ do not intersect $A$. Now for $j$ sufficiently large we have $f_{j}(D) \subset G$. We also have $f_{j}(0)=p$ and $f_{j}^{\prime}(0)=c \xi$.

If we drop the requirement that $f(D) \subset \subset G$, we may still apply the foregoing argument to any mapping of the form $z \mapsto f(r z)$ where $0<r<1$. This gives a map $g: D \rightarrow G \backslash A$ such that $g(0)=p$ and $g^{\prime}(0)=r c \xi$. (We note that $f$ is the uniform limit on compact subsets of maps of the same form as $g$.) It is now easy to see that $K_{G \backslash A}(p ; \xi)=K_{G}(p ; \xi)$.

Remarks 3.15. (1) The condition in Proposition 3.13 is not necessary for the inclusion map to be a Kobayashi isometry at one point. For example, when $G=\Omega$ is a strictly convex bounded domain, we can remove from $\Omega$ any proper closed subset of one extremal disc. The inclusion map is an isometry for the infinitesimal Kobayashi metric at all remaining points of the extremal disc. One cannot remove a subset of $\Omega$ with nonempty interior, however.

Question: Does there exist a hyperbolic domain $G$ from which one can remove a subset $A$ with nonempty interior, such that the inclusion map $i: G \backslash A \rightarrow$ $G$ is a Kobayashi isometry at one point?

(2) Another example of a holomorphic map which is a Kobayashi isometry (everywhere) but fails to be biholomorphic is the following; let $M$ be a hyperbolic complex manifold, not simply connected, let $N$ be its universal covering, and consider the covering projection $\pi: N \rightarrow M$. 
(3) Examples of holomorphic maps which are Carathéodory isometries at one point but fail to be biholomorphic have been given by Vigué [27].

(4) In [22], Pelles showed that a holomorphic map $F$ between hyperbolic complex manifolds which preserves the Eisenman-Kobayashi measure of all sets must be biholomorphic. This assumption cannot be weakened to $F$ preserves the Eisenman-Kobayashi volume form, as the example in Remark (2) shows. Nor need $F$ be biholomorphic if it preserves the Hausdorff measure associated to the Kobayashi distance (examples of Campbell-Ogawa type). Likewise $F$ need not be biholomorphic if it preserves the Hausdorff measure associated to the Carathéodory metric, or the Carathéodory volume form or measure. (For a counterexample to all Carathéodory cases, let $G_{1} \subset G_{2}$ be bounded domains in $\mathbf{C}^{n}$ such that any $f \in H^{\infty}\left(G_{1}\right)$ extends isometrically to $\hat{f} \in H^{\infty}\left(G_{2}\right)$, and consider the inclusion map.) $F$ is locally biholomorphic in all of these cases, however.

(5) A holomorphic self-map of a hyperbolic manifold $M$ with a fixed point $p$ such that $|J f(0)|=1$ must be biholomorphic [16, Chapter V, Theorem 3.3] and [15, Satz 3.6]. Suppose one moves away from self-maps with fixed points, and considers instead a holomorphic map $F: M_{1} \rightarrow M_{2}$ which preserves the Carathéodory volume form $\eta$ or the Eisenman-Kobayashi volume from $\tau$ at one point. Unless $M_{1}=B^{n}$ or $M_{2}=B^{n}$, I do not know of any conditions under which $F$ must be biholomorphic. $B^{n}$ arises because it is used to define both $\eta$ and $\tau$. Thus a holomorphic map $F: M \rightarrow B^{n}$ which preserves $\tau$ at one point is biholomorphic, while a map $F: B^{n} \rightarrow M$ which preserves $\eta$ at one point is biholomorphic. (The latter assertion is easy, while the former is equivalent to [14, Theorem 1]. See also [6].)

\section{A REMARK ON A THEOREM OF BLAND AND GRAHAM}

In this section we give a criterion for the existence of a holomorphic map from $B^{n}$ to a complex manifold $M$ of dimension $n$ which is a Kobayashi isometry at one point. The result improves the hypotheses of [2, Theorem $2(\mathrm{~d})]$.

We recall that $E_{n}\left(p ; \partial / \partial z_{1} \wedge \cdots \wedge \partial / \partial z_{n}\right)$ denotes the Eisenman-Kobayashi volume density in local holomorphic coordinates at $p$, and that $\left(d H_{K}^{2 n} / d \lambda^{2 n}\right)(p)$ denotes the density of the top-dimensional Hausdorff measure associated to the Kobayashi metric.

Theorem 4.1. If $M$ is a taut complex manifold and there exists a point $p \in M$ such that

$$
\frac{d H_{K}^{2 n}}{d \lambda^{2 n}}(p)=E_{n}\left(p ; \frac{\partial}{\partial z_{1}} \wedge \cdots \wedge \frac{\partial}{\partial z_{n}}\right)
$$

then there exists a holomorphic mapping $f: B^{n} \rightarrow M$ such that $f(0)=p$ and $f$ is a Kobayashi isometry at 0 . (The improvement is that convexity of the Kobayashi indicatrix at $p$ need not be assumed.) 
Proof. Let $f: B^{n} \rightarrow M$ be a holomorphic map which assumes the infimum in the definition

$$
\begin{aligned}
E_{n} & \left(p ; \frac{\partial}{\partial z_{1}} \wedge \cdots \wedge \frac{\partial}{\partial z_{n}}\right) \\
& =\inf \left\{\frac{1}{|J f(0)|^{2}} \mid f: B^{n} \rightarrow M \text { holomorphic, } f(0)=p\right\} .
\end{aligned}
$$

Such an $f$ exists because $M$ is taut. Let $I_{K}(p)$ denote the Kobayashi indicatrix at $p$, and let $\widehat{I}_{K}(p)$ denote its convex hull. The Kobayashi indicatrix at the origin of $B^{n}$ we again denote by $B^{n}$. The fact that

$$
d f_{0}\left(B^{n}\right) \subset I_{K}(p) \subset \widehat{I}_{K}(p)
$$

gives

$$
\frac{1}{|J f(0)|^{2}} \geq \frac{\operatorname{vol}\left(B^{n}\right)}{\operatorname{vol}\left(I_{K}(p)\right)} \geq \frac{\operatorname{vol}\left(B^{n}\right)}{\operatorname{vol}\left(\widehat{I}_{K}(p)\right)}
$$

(vol denotes Euclidean volume in local coordinates). On the other hand the formula (see [2])

$$
\frac{d H_{K}^{2 n}}{d \lambda^{2 n}}(p)=\frac{\operatorname{vol}\left(B^{n}\right)}{\operatorname{vol}\left(\widehat{I}_{K}(p)\right)}
$$

together with the assumption of the theorem gives

$$
\frac{1}{|J f(0)|^{2}}=\frac{\operatorname{vol}\left(B^{n}\right)}{\operatorname{vol}\left(\widehat{I}_{K}(p)\right)} \text {. }
$$

This shows that equality occurs at each stage in (4.2). Since $I_{K}(p)$ has continuous boundary under the assumption of tautness of $M$, we must have

$$
d f_{0}\left(B_{n}\right)=I_{K}(p)=\widehat{I}_{K}(p) .
$$

This shows that $I_{K}(p)$ is indeed convex, and that $d f_{0}$ is a Kobayashi isometry.

\section{A Remark on a theORem of Fornaess ANd Sibony}

The following is a result of Fornaess and Sibony [8, Theorem 3.2], except that we have replaced a hypothesis on the Kobayashi metric by a hypothesis on the Eisenman-Kobayashi volume form $\tau$ :

Theorem 5.1. Suppose that $M$ is a complex manifold which is an increasing union of biholomorphic images of a fixed complex manifold $\Omega$. Suppose that $\Omega$ is hyperbolic and that $\Omega /$ Aut $\Omega$ is compact. If there exists a point $p \in M$ such that $\tau(p) \neq 0$ then $M$ is biholomorphic to $\Omega$.

The original assumption of Fornaess and Sibony was that there should exist a point $p \in M$ such that $K(p ; \xi) \neq 0$ for all $\xi \in T_{p} M \backslash\{0\}$. Since the purpose of the assumption is to show that a certain mapping is nondegenerate, an assumption on $\tau$ seems more natural as well as slightly more general. 
Proof. To prove this generalization it suffices to derive an analog of [8, Lemma 3.1] for intrinsic volume forms. We shall use the notation of that paper in this section. Thus $\Omega$ is no longer a convex domain in $\mathbf{C}^{n}$, but a hyperbolic complex manifold such that $\Omega /$ Aut $\Omega$ is compact. (This assumption implies that $\Omega$ is taut, in fact complete hyperbolic [8, Lemma 2.1].) The complex manifold $M$ can be written

$$
M=\bigcup_{k=1}^{\infty} M_{k} \supset \cdots \supset M_{k} \supset \cdots \supset M_{2} \supset M_{1}
$$

where each $M_{k}$ is biholomorphic to $\Omega$. Since there is a compact subset $K$ of $\Omega$ such that $\Omega=($ Aut $\Omega) \cdot K$, we can find a point $p_{0} \in M_{1}$ and biholomorphic maps $\phi_{k}: \Omega \rightarrow M_{k}$ such that $\phi_{k}^{-1}\left(p_{0}\right)=q_{k} \in K$. Let $\psi_{k}=\phi_{k}^{-1}$. The tautness of $\Omega$ gives a subsequence of the $\left\{\psi_{k}\right\}$, which we again denote by $\left\{\psi_{k}\right\}$, and a holomorphic map $\psi: M \rightarrow \Omega$ such that $\psi_{k} \rightarrow \psi$ uniformly on compact subsets of $M$. We have

Lemma 5.2. $\tau_{M}=\psi^{*}\left(\tau_{\Omega}\right)$.

Proof. Let $p \in M$ and let $\beta \in \Lambda^{n} T_{p} M$. We wish to show that

$$
E_{n}^{M}(p ; \beta)=E_{n}^{\Omega}\left(\psi(p) ; \psi_{*}(\beta)\right) .
$$

Evidently

$$
E_{n}^{M_{k}}(p ; \beta)=E_{n}^{\Omega}\left(\psi_{k}(p),\left(\psi_{k}\right)_{*}(\beta)\right)
$$

whenever $k$ is large enough that $p \in \phi_{k}(\Omega)$, since $\psi_{k}$ is a biholomorphic map. Since $M_{k} \nearrow M, E_{n}^{M_{k}}(p ; \beta) \searrow E_{n}^{M}(p ; \beta)$. Now since $\Omega$ is taut,

$$
E_{n}^{\Omega}\left(\psi(p) ; \psi_{*}(\beta)\right) \leq \varliminf_{k \rightarrow \infty} E_{n}^{\Omega}\left(\psi_{k}(p),\left(\psi_{k}\right)_{*}(\beta)\right) .
$$

On the other hand, $E_{n}^{\Omega}$ is upper semicontinuous on $\Lambda^{n} T \Omega$ by a result of Pelles [22, Lemma 2.5]. (Pelles actually shows that if we choose local coordinates $z_{1}, \ldots, z_{n}$ near $q \in \Omega$, the function $E_{n}^{\Omega}\left(q ; \partial / \partial z_{1} \wedge \cdots \wedge \partial / \partial z_{n}\right)$ is upper semicontinuous in $q$. However since $E_{n}^{\Omega}(q ; \gamma)$ is homogeneous of degree $2 n$ in $\gamma$, Pelles' result easily gives the more general statement.) This gives

$$
E_{n}^{\Omega}\left(\psi(p), \psi_{*}(\beta)\right) \geq \varlimsup_{k \rightarrow \infty} E_{n}^{M_{k}}(p ; \beta)=E_{n}^{M}(p ; \beta)
$$

as desired.

Lemma 5.2 and the assumption of Theorem 5.1 imply that there is a point $p \in M$ at which $d \psi$ is invertible. The rest of the argument is the same as in [8, Theorem 3.2].

\section{MeASURE-HYPERBolic COMPLETENESS}

We recall that a domain $G \varsubsetneqq \mathbf{C}^{n}$ is measure-hyperbolically complete at a point $p \in \partial G$ if whenever $U$ is a neighborhood of $p$ we have $\int_{U \cap G} \tau_{G}=$ $\infty$. Evidently the set of points in $\partial G$ at which $G$ is measure-hyperbolically 
complete is closed. $G$ itself is measure-hyperbolically complete if it is complete at all boundary points.

In [13] it is asked whether a measure-hyperbolically complete domain is a domain of holomorphy. In this section we shall answer the question in the negative by constructing a counterexample.

Let $D_{r}=\{z \in \mathbf{C}|| z \mid<r\}$. We shall denote the unit disc interchangeably by $D$ and $D_{1}$. We claim that the domain $G=\left(D_{1} \times D_{2}\right) \cup\left(D_{2} \times D_{1}\right)$ in $\mathbf{C}^{2}$ is measure-hyperbolically complete. Since it fails to be logarithmically convex it is not a domain of holomorphy.

Since $G \subset D_{2} \times D_{2}$ and since $\tau_{D_{2} \times D_{2}}=\tau_{D_{2}} \wedge \tau_{D_{2}}[13, \S 3], G$ is measurehyperbolically complete at all common boundary points of $G$ and $D_{2} \times D_{2}$. It suffices to show that $G$ is measure-hyperbolically complete at boundary points of the form $\left(p_{1}, e^{i \theta}\right)$ where $\left|p_{1}\right|>1$. We shall therefore estimate $\tau$ at points $\left(p_{1}, p_{2}\right)$ of $G$ with $\left|p_{1}\right|>1$. Let $f: B^{2} \rightarrow G$ be a holomorphic map such that $f(0)=p$. We wish to give a lower bound for $|J f(0)|^{-2}$. Let $f=\left(f_{1}, f_{2}\right)$. By applying a unitary transformation of $B^{2}$ if necessary, we may assume that $\left(\partial f_{1} / \partial z_{2}\right)(0)=0$. Hence

$$
J f(0)=\frac{\partial f_{1}}{\partial z_{1}}(0,0) \cdot \frac{\partial f_{2}}{\partial z_{2}}(0,0) .
$$

By applying the Schwarz lemma to $f_{1}(z, 0)$ we obtain $\left(\partial f_{1} / \partial z_{1}\right)(0,0) \leq$ $\frac{1}{2}\left(4-\left|p_{1}\right|^{2}\right)$.

The remainder of the argument amounts to estimating the Kobayashi metric in the normal direction and is based on [13, Example 6.4]. Define $\phi: D \rightarrow G$ by $\phi(z)=f(0, z)$ and write $\phi=\left(\phi_{1}, \phi_{2}\right)$. There are two cases to consider:

Case 1: $\phi_{2}(D) \subset D$. In this case the Schwarz lemma gives $\left|\phi_{2}^{\prime}(0)\right| \leq 1-\left|p_{2}\right|^{2}$.

Case 2: $\phi_{2}(D) \not \subset D$. In this case we may choose $r \in D$ of minimal modulus such that $\left|\phi_{2}(r)\right|=1$. We may also assume $r>0$. Then $\phi_{2}\left(D_{r}\right) \subset D$, so the Schwarz lemma gives $r\left|\phi_{2}^{\prime}(0)\right| \leq 1-\left|p_{2}\right|^{2}$. We can find a lower bound for $r$ by considering $\phi_{1}$, using the fact that $\left|\phi_{1}(r)\right|<1$ while $\left|\phi_{1}(0)\right|=\left|p_{1}\right|>1$. We have

$$
\phi_{1}(r)-\phi_{1}(0)=\int_{0}^{1} \frac{d}{d t} \phi_{1}(r t) d t=\int_{0}^{1} r \phi_{1}^{\prime}(r t) d t
$$

which implies

$$
\left|p_{1}\right|-1<\left|\phi_{1}(r)-\phi_{1}(0)\right| \leq r \int_{0}^{1}\left|\phi_{1}^{\prime}(r t)\right| d t .
$$

From this we can conclude that there exists a number $s, 0<s<r$, such that $\left|\phi_{1}^{\prime}(s)\right|>r^{-1}\left(\left|p_{1}\right|-1\right)$. Applying the Schwarz lemma to $\phi_{1}$ at $s$ now gives

$$
\left|\phi_{1}^{\prime}(s)\right| \leq \frac{1}{2} \cdot \frac{4-\left|\phi_{1}(s)\right|^{2}}{1-s^{2}}<\frac{2}{1-r^{2}} .
$$


Together with the lower bound for $\left|\phi_{1}^{\prime}(s)\right|$ this gives

$$
r^{2}\left(\left|p_{1}\right|-1\right)+2 r-\left(\left|p_{1}\right|-1\right)>0 .
$$

Since $r>0$ we obtain

$$
r>\frac{-1+\sqrt{1+\left(\left|p_{1}\right|-1\right)^{2}}}{\left|p_{1}\right|-1}=\frac{\left|p_{1}\right|-1}{1+\sqrt{1+\left(\left|p_{1}\right|-1\right)^{2}}}>\frac{1}{2}\left(\left|p_{1}\right|-1\right) .
$$

Thus in case 2 we have

$$
\left|\phi_{2}^{\prime}(0)\right|<\frac{2\left(1-\left|p_{2}\right|^{2}\right)}{\left|p_{1}\right|-1},
$$

an estimate which is evidently weaker than case 1 . In either case we have

$$
\frac{1}{|J f(0)|^{2}}>\frac{\left(\left|p_{1}\right|-1\right)^{2}}{\left(4-\left|p_{1}\right|^{2}\right)^{2}\left(1-\left|p_{2}\right|^{2}\right)^{2}}
$$

Thus

$$
E_{2}^{G}\left(p, \frac{\partial}{\partial z_{1}} \wedge \frac{\partial}{\partial z_{2}}\right)>\left(\left|p_{1}\right|-1\right)^{2} E_{2}^{D_{2} \times D_{1}}\left(p, \frac{\partial}{\partial z_{1}} \wedge \frac{\partial}{\partial z_{2}}\right) .
$$

This estimate shows that $G$ is measure-hyperbolically complete at $\left(p_{1}, e^{i \theta}\right)$. Hence we are done.

\section{REFERENCES}

1. T. Barth, Taut and tight complex manifolds, Proc. Amer. Math. Soc. 24 (1970), 429-431.

2. J. Bland and I. Graham, On the Hausdorff measure associated to the Carathéodory and Kobayashi metrics, Ann. Scuola Norm. Sup. Pisa 12 (1985), 503-514.

3. D. Burns and S. Krantz (to appear)

4. L. A. Campbell, A. Howard and T. Ochiai, Moving holomorphic disks off analytic subsets, Proc. Amer. Math. Soc. 60 (1976), 106-108.

5. L. A. Campbell and R. H. Ogawa, On preserving the Kobayashi pseudodistance, Nagoya Math. J. 57 (1975), 37-47.

6. I. M. Dektyarev, Tests for equivalence of hyperbolic manifolds, Funktsional Anal. i Prilozhen. 15 (1981), 73-74. (English transl., Funct. Anal. Appl. 15 (1981), 292-293.)

7. D. A. Eisenman (=D. A. Pelles), Intrinsic measures on complex manifolds and holomorphic mappings, Mem. Amer. Math. Soc. No. 96 (1970).

8. J. E. Fornaess and N. Sibony, Increasing sequences of complex manifolds, Math. Ann. 255 (1981), 351-360.

9. J. E. Fornaess and E. L. Stout, Polydiscs in complex manifolds, Math. Ann. 227 (1977), 145-153.

10. I. Graham, Intrinsic measures on complex manifolds, Mat. Vesnik 38 (1986), 475-488.

11. __ Intrinsic measures and holomorphic retracts, Pacific J. Math. 130 (1987), 299-311.

12. Holomorphic mappings into strictly convex domains which are Kobayashi isometries at one point, Proc. Amer. Math. Soc. 105 (1989), 917-921.

13. I. Graham and $\mathrm{H}$. Wu, Some remarks on the intrinsic measures of Eisenman, Trans. Amer. Math. Soc. 288 (1985), 625-660.

14. Characterizations of the unit ball $B^{n}$ in complex Euclidean space, Math. Z. 189 (1985), 449-456. 
15. W. Kaup, Transformationengruppen und invariante Metriken, Invent. Math. 3 (1967), 4370.

16. S. Kobayashi, Hyperbolic manifolds and holomorphic mappings, Dekker, New York, 1970.

17. _ Intrinsic distances, measures, and geometric function theory, Bull. Amer. Math. Soc. 82 (1976), 357-416.

18. L. Lempert, La métrique de Kobayashi et la representation des domaines sur la boule, Bull. Soc. Math. France 109 (1981), 427-474.

19. $\ldots$, Holomorphic retracts and intrinsic metrics in convex domains, Anal. Math. 8 (1982), 257-261.

20. __ Intrinsic distances and holomorphic retracts, Complex Analysis and Applications 1981, Sofia (1984), pp. 341-364.

21. G. Patrizio, On holomorphic maps between domains in $\mathbf{C}^{n}$, Ann. Scuola Norm. Sup. Pisa 13 (1986), 267-279.

22. D. A. Pelles (=D.A. Eisenman), Holomorphic maps which preserve intrinsic measure, Amer. J. Math. 97 (1975), 1-15.

23. H. L. Royden, Remarks on the Kobayashi metric. Several complex variables. II (Proc. Internat. Conf. Univ. of Maryland, 1970), Lecture Notes in Math., vol. 185, Springer-Verlag, New York-Heidelberg-Berlin, 1971, pp. 125-137.

24. H. L. Royden and P. M. Wong, Carathéodory and Kobayashi metrics on convex domains, preprint.

25. E. Vesentini, Complex geodesics, Compositio Math. 44 (1981), 375-394.

26. Complex geodesics and holomorphic maps, Symposia Math. 26 (1982), 211-230.

27. J. P. Vigué, Caracterisation des automorphismes analytiques d'un domaine convexe borné, C. R. Acad. Sci. Paris 299 (1984), 101-104.

28. __ Sur la caractérisation des automorphismes analytiques d'un domaine borné, Portugal. Math. 43 (1985-1986), 439-453.

29. H. Wu, Normal families of holomorphic mappings, Acta Math. 119 (1967), 194-233.

Department of Mathematics, University of Toronto, Toronto, Canada M5S 1A1

Department of Mathematics, Stanford University, Stanford, California 94305 